\title{
Modelling and Analysis of IPTV Usage Patterns for Improving Quality of Service
}

\author{
P. L. Srinivasa Murthy \\ Associate Professor, \\ Gokaraju Rangaraju \\ Institute of Engineering \& Technology, \\ Hyderabad, India.
}

\author{
T. Venu Gopal, PhD \\ Associate Professor, \\ JNTUH College of Engineering, \\ Sultanpur, \\ Hyderabad, India.
}

\begin{abstract}
Of late IPTV usage is growing rapidly as the viewers are interested to watch stored videos interactively over IP. The bursts in user demands for on-demand content can cause unexpected burden on the content dissemination infrastructure. Stated differently the usage dynamics of video content has its impact on the responsiveness, bandwidth and server. Especially it is non-trivial to solve the problem when number of subscribers is very huge. Here comes the need for modelling and the usage patterns of IPTV and analysing it for making important strategies for server to cope with bursts of subscriber requests. The discovery of usage patterns also considers periods of usage such as week day and week end. In this paper a framework is proposed that can help in modelling and analysis of IPTV usage patterns. The video streaming control events are also considered for the modelling. Characterization of stream control events using a finite state machine with and estimated Markov chain is made. The proposed modelling is validated with traces of operational IPTV environment in large scale.
\end{abstract}

\section{Keywords}

IPTV, modeling and analysis, finite state machine, markov chain

\section{INTRODUCTION}

Of late, users are interested to watch videos on demand (VoD) interactively. In fact users have more control over the streaming content as they can watch it interactively. When number of interactive users is increased and there are sudden bursts in the requests, it can cause burden on the content dissemination infrastructure such as set top boxes, network and servers. When the stream is controlled by the viewers, the control operations can have impact on the responsiveness of the system. When the system is not able to cope with the pressure of interactive video streaming its performance will be deteriorated. It finally results in poor customer satisfaction. In spite of sudden increase in demand and interactive nature of users for watching videos, it is essential that the server provider has to ensure minimum responsiveness. The technical knowhow on the users' behaviour with respect to stream control events, as shown in Figure 1, can help service providers to optimize their system to deliver video content with reasonable quality. The optimization includes the bandwidth, number of servers, processing capability of set-top boxes and good mechanisms to delivery video content.

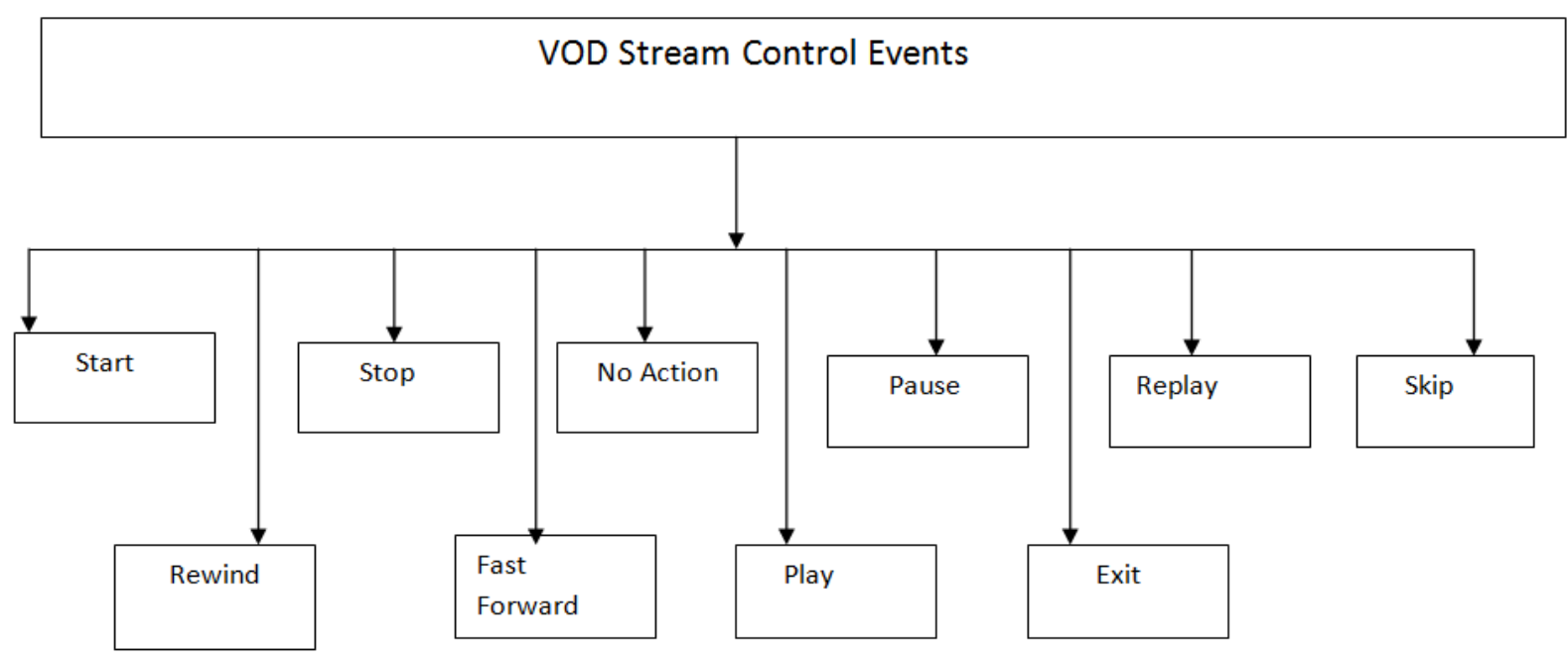

Figure 1 - VoD Stream control events

The stream control events are self descriptive in nature. In order to handle these events it is important to have buffering capabilities besides fine-tuning timers for quality content delivery. To ensure quality of services the service providers need to have intelligence about interactive behaviour of users.
This will help service providers to take well informed decisions. In this paper a framework is proposed that can help in modelling user behavioural patterns with respect to the interactive usage of IPTV. The contributions of this paper are as follows. 
The interactive user behavior is modeled while accessing IPTV for VoD. The model focuses on two aspects of video streaming. The first one is modelling the arrival process of streaming while the second one is modelling the usage model. These two distinctive models are used for characterization of VoD stream with interactive users.

The stream control model is to model the stream control events in the form of finite state machine (FSM). Each state in the FSM represents a stream control event.

The remainder of the paper is structured as follows. Section II reviews literature on the relevant topics. Section III provides the proposed model for characterising user behaviour with respect to interactive $\mathrm{VoD}$ streaming. Section IV provides results while section $\mathrm{V}$ concludes the paper and provides future work.

\section{RELATED WORKS}

Modeling user activities pertaining to interactive viewing of IPTV can help service providers to have best strategies to deal with the users with reasonable responsiveness. Towards this end many researchers contributed. Qiu et al. [1] explored IPTV and modelled user activities. They built a prototype application named SimulWatch to demonstrate the modelling of user behaviour. Lin et al. [2] proposed an expectation confirmation model for finding the user's IPTV continuance intention. This will help the service provider to make necessary actions and improve customer satisfaction. Houwman et al. [3] proposed a business model for IPTV services. They focused on the service provider and emergence of new service providers in the market of IPTV. The behaviour of IPTV users was focused for the purpose of gaining business intelligence. In [4] there is research on the interactive and personalized IPTV. They proposed an adaptive framework that can provide rich experience to end users.

Shin et al. [5] explored the possibility of analysing Mobil IPTV and the factors that influence the consumer adaptation. Entertainment and emotional factors were focused and they can help to understand user behaviour with respect to IPTV. Choi et al. [6] explored personalized and web based IPTV services that can help users to have to have convenience. Their study focuses on the architectural issues for building a viable platform for ensuring a truly personalized IPTV service. Shin [7] studied the factors that influence the adaptation of IPTV besides finding customer expectations. The user factors found can help service providers to improve quality of services. The potential user factors that influence the adaptation of IPTV include intrinsic factors, extrinsic features, cost, and demographics. Kim and Kang [8] explored the possibility of personalized advertisement. The personalized advertisement can help organizations to target customer behaviour. The solution is ontology based where knowledge is represented in the form of concepts and the relationships among the concepts.
Zajc et al. [9] explored the challenges of interactive digital television where the users can have flexibility of operating the channels. The researchers presented architecture for content flow with respect to providing IPTV services. On -demand IPTV services can play vital role in the newly formed environment as well. Diminico et al. [10] studied long term usage patterns of IPTV. Thus the researchers could gain the possible insights into the capacity requirements of IPTV services that are to be rendered. Thus long term usage patterns are recorded in order to have useful knowledge pertaining to capacity requirements. Silverston et al. [11] explored the traffic analysis of IPTV communities and that run on Peer 2 Peer network. The traffic analysis can help improve the quality of services in IPTV network. The work in [12] is similar to that of [1]. Riede et al. [13] proposed an IPTV architecture that addresses issues like interoperability, interactivity and integration. Noem et al. [14] explored Internet Television. Their research was focused on the business models that have been Television over the Internet. Junior et al. [15] explored Java interactive television (JiTV) which is a platform that can be used to have multimedia content dissemination.

Lee et al. [16] explored distributed pre-recorded IPTV content exploited the concept of performance aware replication. The authors proposed a framework known as Smart Personal Information Network (Smart PIN) which is best used for performance based dissemination of content in the real world. Ma et al. [17] proposed and implemented a model that takes care of usage of IPTV on the pull model of P2P networks. Began et al. [18] explored the possibility of isolating faults in IPTV which helped them to reduce the faults and ensure quality of IPTV service to end users. Kampmann [19] explored the process of prediction of IPTV usage. This is achieving by using different research hypotheses such as intention to use IPTV, expected outcomes with respect to the intention to use IPTV. Shin et al. [20] focused on the interactivity of IPTV. However, Shin et al. explored the effects of activities such as continuous use intention, emotion and trust. All these efforts were made to know customer behaviour with respect to IPTV and make strategies to provide quality of services and achieve higher customer satisfaction. In this paper a framework is proposed that caters to the needs of processing IPTV request come from interactive users.

\section{CHARACTERIZATION OF IPTV USAGE PATTERNS}

This section starts with preliminaries that cover the FSM of user behaviour of interactive IPTV, periods of use and different kinds of modelling prior to the framework proposed. The FSM shown in Figure 2 reflects different types of streaming control events with states and transitions among them. 


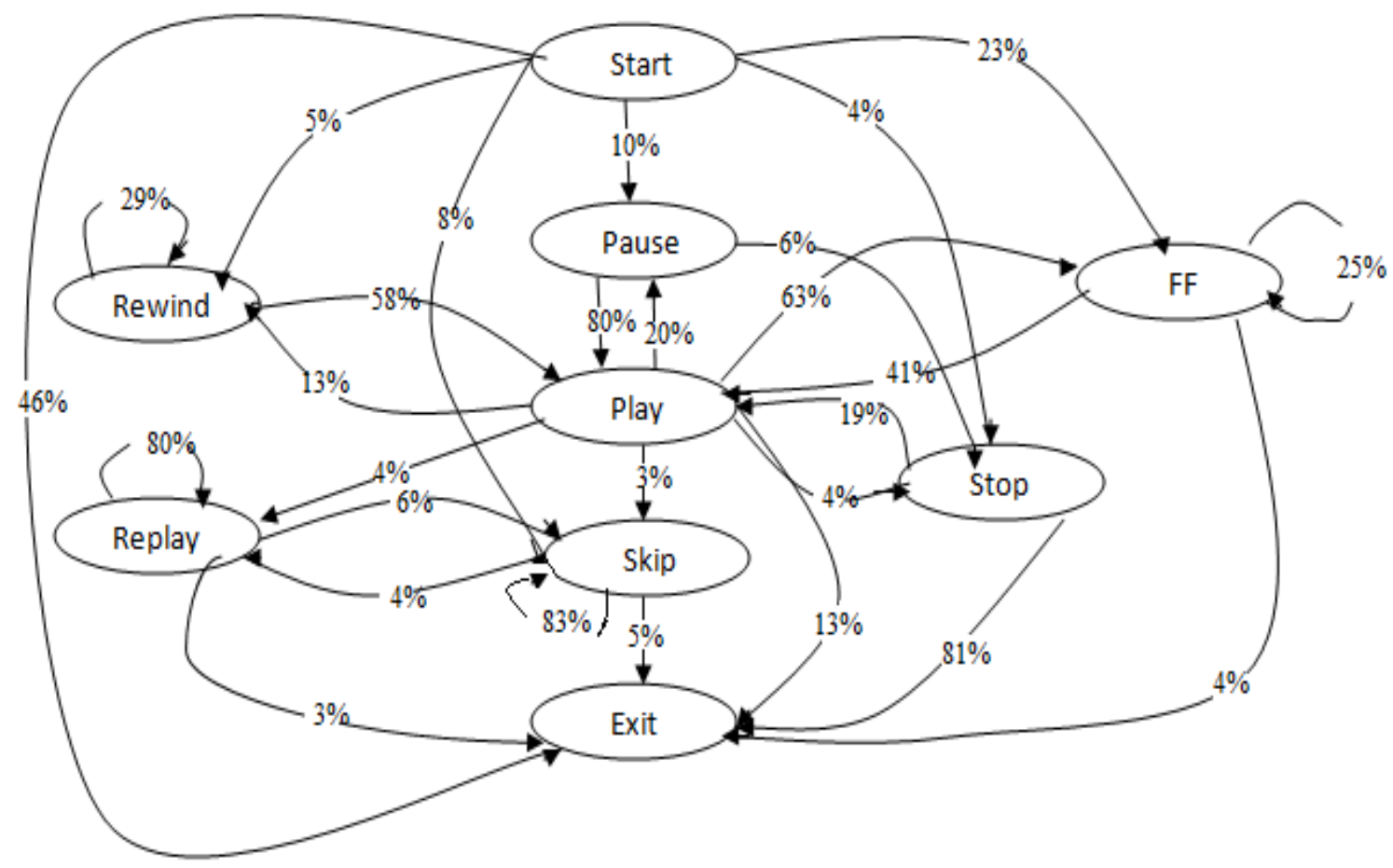

Figure 2 - Finite State Machine (FSM) representing the user behaviour patterns in the week end period

All possible streaming control events are covered in Figure 2. Each event is considered to have a state and there is state transition that reflects the true behaviour of users who watch videos interactively and make use of all streaming control events. The stream control events are stop, no action, pause, replay, skip, rewind, fast forward, play, exit and start.

\subsection{Periods of Usage}

IPTV services are rendered by respective service providers. The service providers are interested to know the behaviour of users with respect to their behaviour on interactive viewing of
IPTV. When users are involved in this work, while modeling the IPTV usage of interactive users two periods of usage are considered. They are known as weekend and weekday as shown in Figure 3. As people are understood to have leisure in the weekend, it is interesting to know if they are likely spend more time with IPTV VoD and how they behave with respect to different stream control events like Pause, Fast Forward, Play and so on.

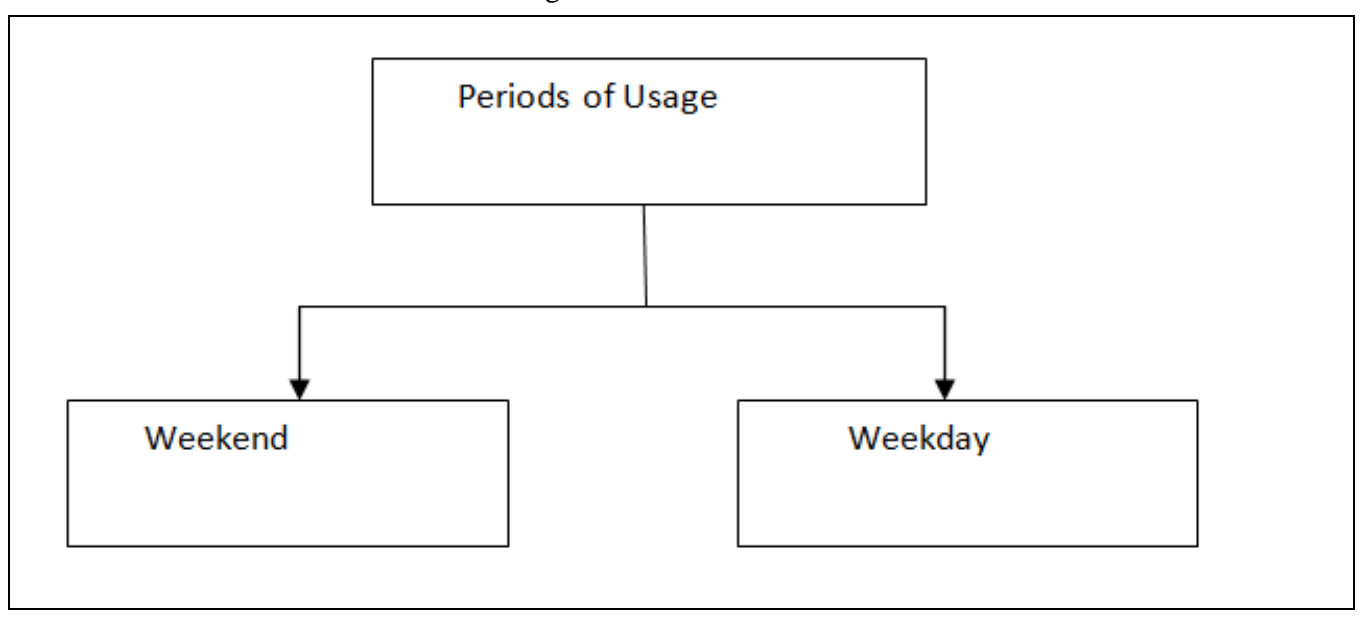

Figure 3 - Periods of usage

As shown in Figure 3, the decision to make use of periods like weekend and weekday is in the wake of expected differences in the behaviour of IPTV users in the real world. As the behavior is likely to differ, the classification of the time into two periods is justified. More realistic statistics with respect to the users' streaming control events can be captured by dividing the time into two periods. 


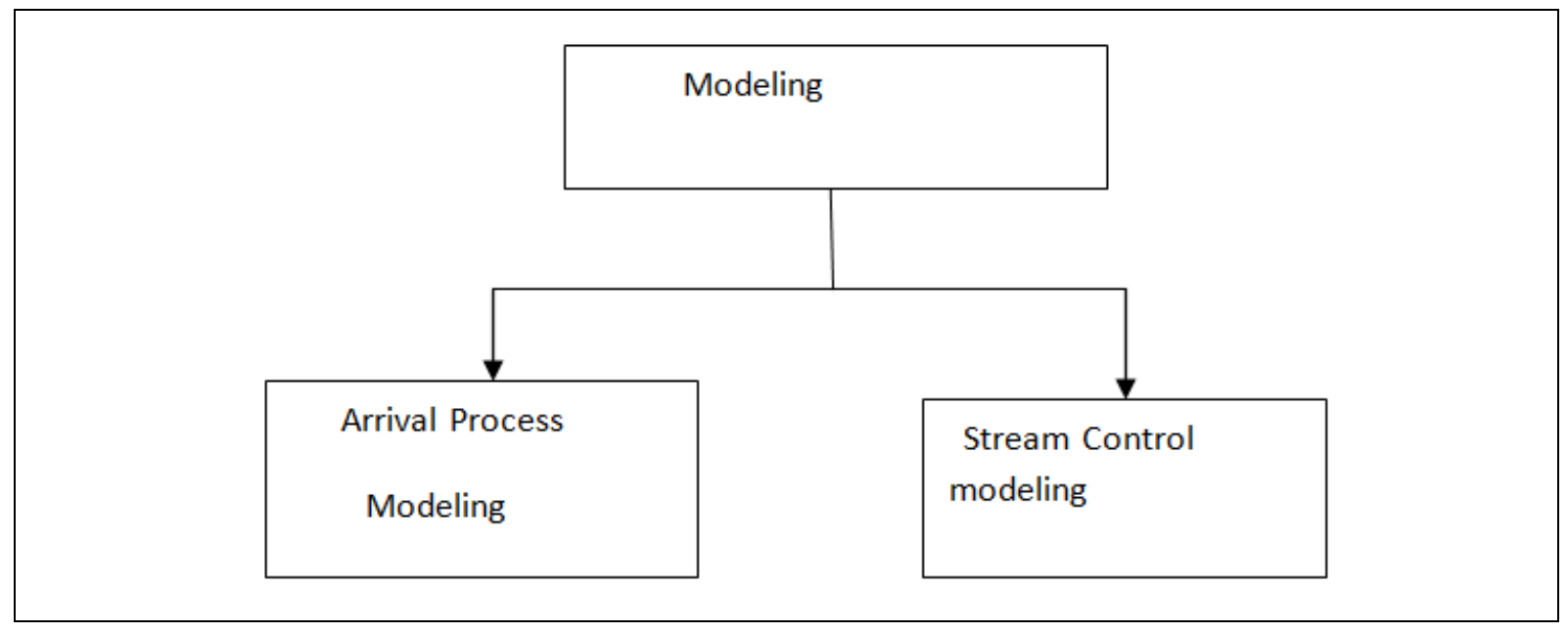

\section{Figure 4 - Modelling approaches}

As shown in Figure 4, two modelling approaches are considered. They are known as modelling of request arrival process at server and the modelling of streaming control events. Both models can help understand users' behaviour with respect to streaming control interactively and also the bursts of requests coming to $\mathrm{VoD}$ server

\subsection{Proposed Framework}

A framework is proposed for modeling and analysis of IPTV usage patterns. The modelling is done in terms of arrival process model and stream control model. The former is to know the request dynamics for $\mathrm{VoD}$ while the latter is to know behaviour of users of IPTV. The arrival process model is based on the data available for two years. The data is collected from a live environment. The arrival process is also modelled for both week end and weekday. The aggregate arrival process is created with respect to VoD. Each arrival of request is converted into a session that reflects a single user performing multiple stream control events. With respect to steam control model, two models are built. These models make use of a common finite state machine (FSM) for modelling sequence of events pertaining to streaming control. This potentially reflects the users' behaviour at run time. The two models are different in the aspect of visit time. The first model the visit time is considered to be independent. This model is known as independent visit time (IVT). The first model is also known as semi-Markov model which can be represented as follows.

\section{$\mathrm{VM}=\mathrm{FSM}+\mathrm{IVT}$}

In the second model the visit time is restricted to have aggregate session and the session duration is distributed. The second model is known as restricted visit time (RVT) model. The two years IPTV streaming data available is used for experiments. The data is collected from live scenario with large number of subscribers. The data reflects variability in VoD that reflects all stream control events and the dynamics of users' behaviour. Thus the data is valuable in order to capture the user behaviour. The user behaviour analysis can bring about business intelligence to service providers. In order characterize the process of arrival of users' requests approximately 120 days data is observed. The users' stream control operations are characterized to reflect steam control operations. The traces are used that cover the live IPTV usage of users. As shown in Figure 2, there are streaming events and state transitions from one state to another state. Both the synthetic traces and real traces are used for experiments.

With respect to VoD usage, the subscribers of IPTV get stream control events as shown in Figure 1, a request is sent to respective server to perform user intended action. When such actions are analyzed and understood, it is easy to model VoD usage in a best way. 


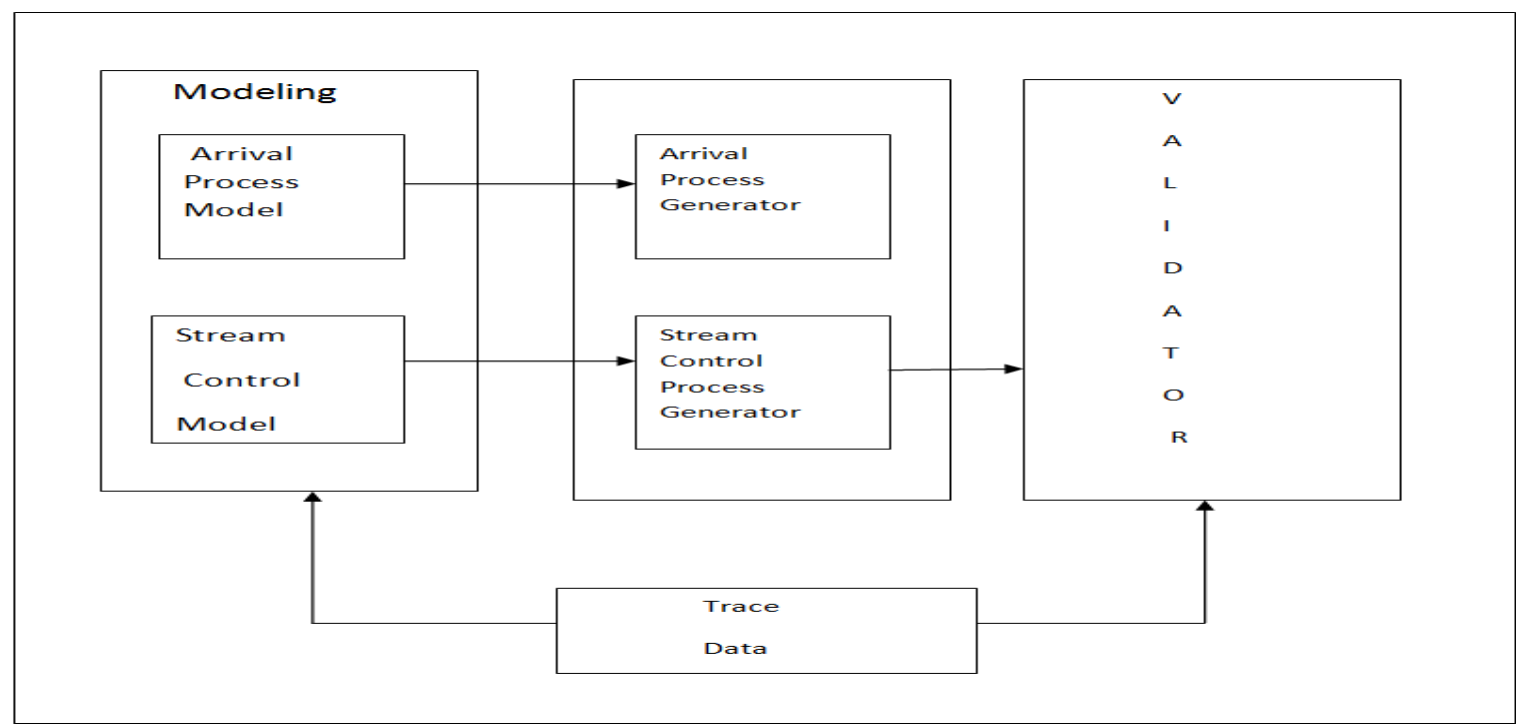

Figure 5 - Proposed framework for analyzing user behaviour patterns of IPTV usage

The user behavior with respect to streaming control events and the influence of the length of the video being rendered are analyzed. The arrival process model shown in the framework is for modeling request arrival at $\mathrm{VoD}$ server. This module is responsible to keep track of requests coming from different sources. It helps in stream control session generation. When number of requests comes from a particular user, it is known as a session. The stream control model is to keep track of stream control events such as stop, no action, pause, replay, skip, rewind, fast forward, play, exit and start. These events are understood by the module and the stream event dynamics are recorded. This will provide very valuable information to service providers as it can bestow knowledge on user behaviour. When user behaviour is known, it is possible to have strategies that can be used to fulfil the requirements of user by ensuring quality of services. The stream control process generator is aware of the movie length distribution. The arrival process generator module is used to have arrival process. A counting mechanism is associated with counting process. The trace data is the basis for the modelling of the arrival and stream control operations. The validator module is responsible to validate the modelling with that of actual work load experienced in the system.

\section{Algorithm for Estimation of Arrival Process}

Algorithm: Estimation of Arrival Process
Inputs: Arrival time of nth customer, number of days D, \# of power events
Output: FFT coefficients and top k FFT coefficients
01 For each d in D
02 for each second normalize count
$03 \quad$ Normalized count is computed
04 End For
05 Compute average normalized count for all days
06 Compute average FFT for each day
07 Find top k FFT coefficients
08 Inverse FFT for regenerating arrival counts
09 return arrival process statistics like FFT coefficients and top k FFT coefficients

The algorithm for estimation of arrival process takes arrival time of many customers, number of days and number of power events. Its output is FFT coefficients and top k FFT coefficients that are used to understand the arrival dynamics. The arrival model of the framework simulates the arrival of requests into the system. The stream control model is used to simulate the stream control events and model them. It makes use of FSM which contains different states and transitions. The states and transitions when tracked can provide the usage behaviour of customers. Thus the proposed system is able to provide the user behaviour statistics. 


\section{EXPERIMENTAL RESULTS}

Experiments are made with real and synthetic traces that provide the details of video streaming and the users' behaviour with respect to interactive stream control events. The observations are made with movie length distribution in terms of movie length and cumulative distribution function (CDF). Then movie length distribution is examined with week day and week end. The rationale behind this is that the subscribers of IPTV are expected to have different behaviour in week end and week day. The behaviour of subscriber is determined in terms of stream control events. The arrival process is captured using the proposed algorithm.

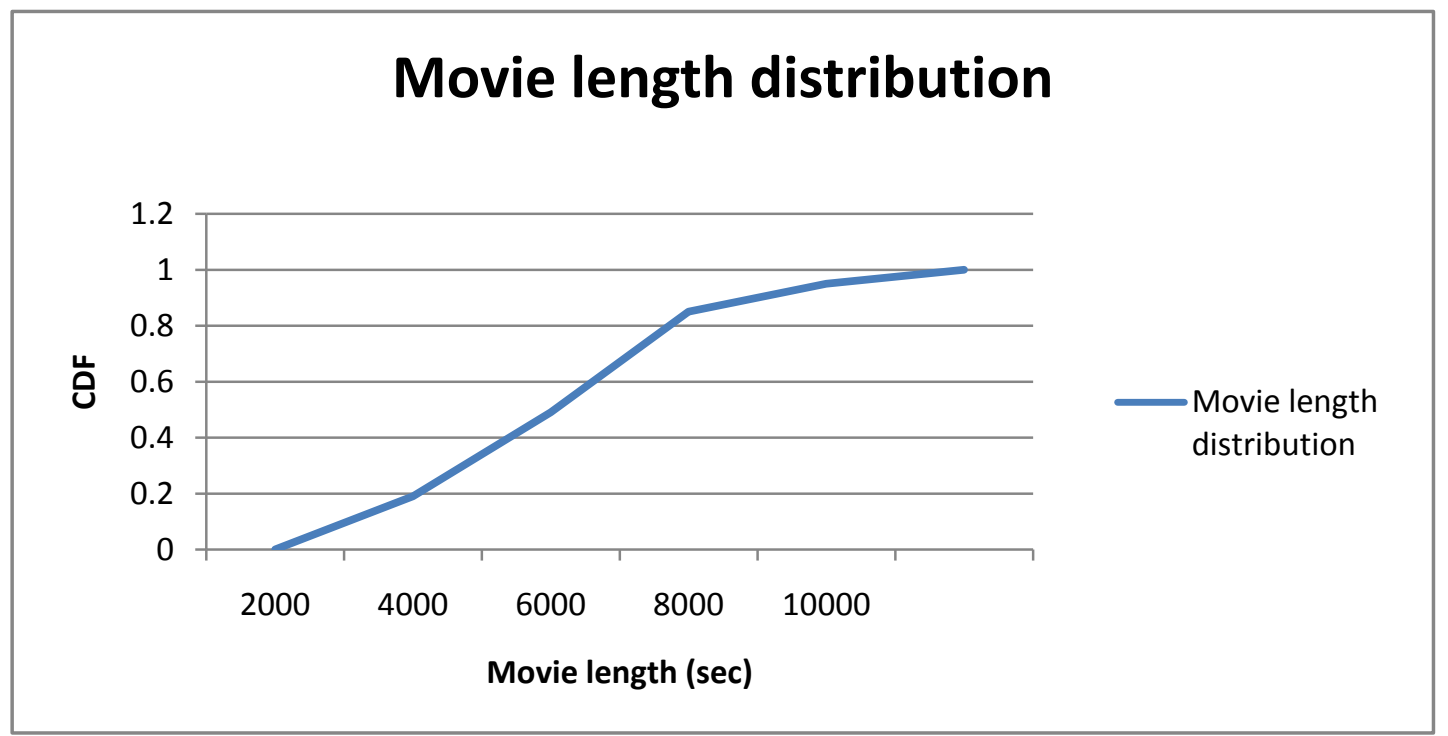

Figure 6 - Movie length distribution in seconds vs. CDF

As shown in Figure 6, the movie length distribution is presented with corresponding CDF dynamics encountered. Movie length is taken in seconds. Movie length is represented by horizontal axis while the vertical axis represents CDF. The $\mathrm{CDF}$ is gradually increased as the movie length is increased.

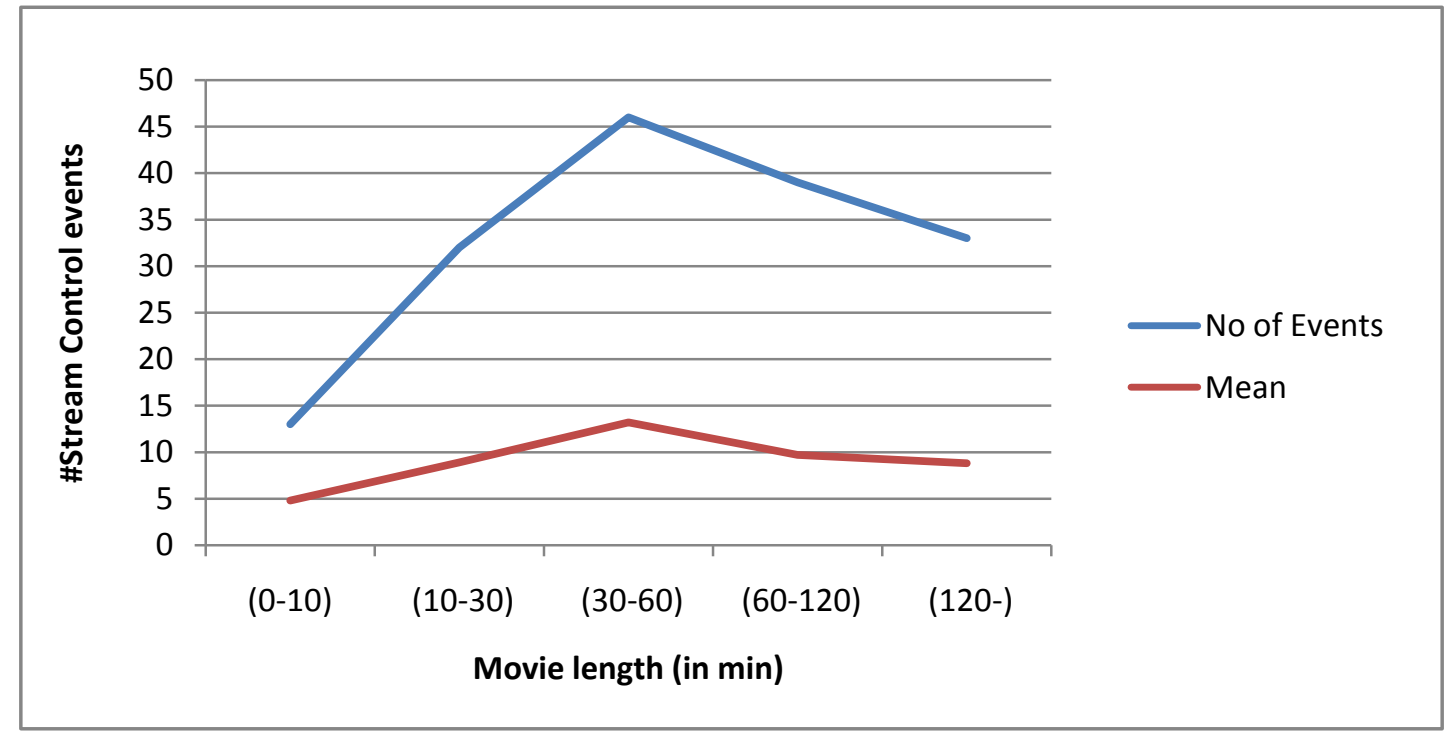

Figure 7 - \# of events for a session vs. movie length (Weekend)

As shown in Figure 7, it is evident that the number of events in user session by movie length. The results revealed that the number of events changed as movie progresses. These results are captured from the weekend usage of IPTV. 


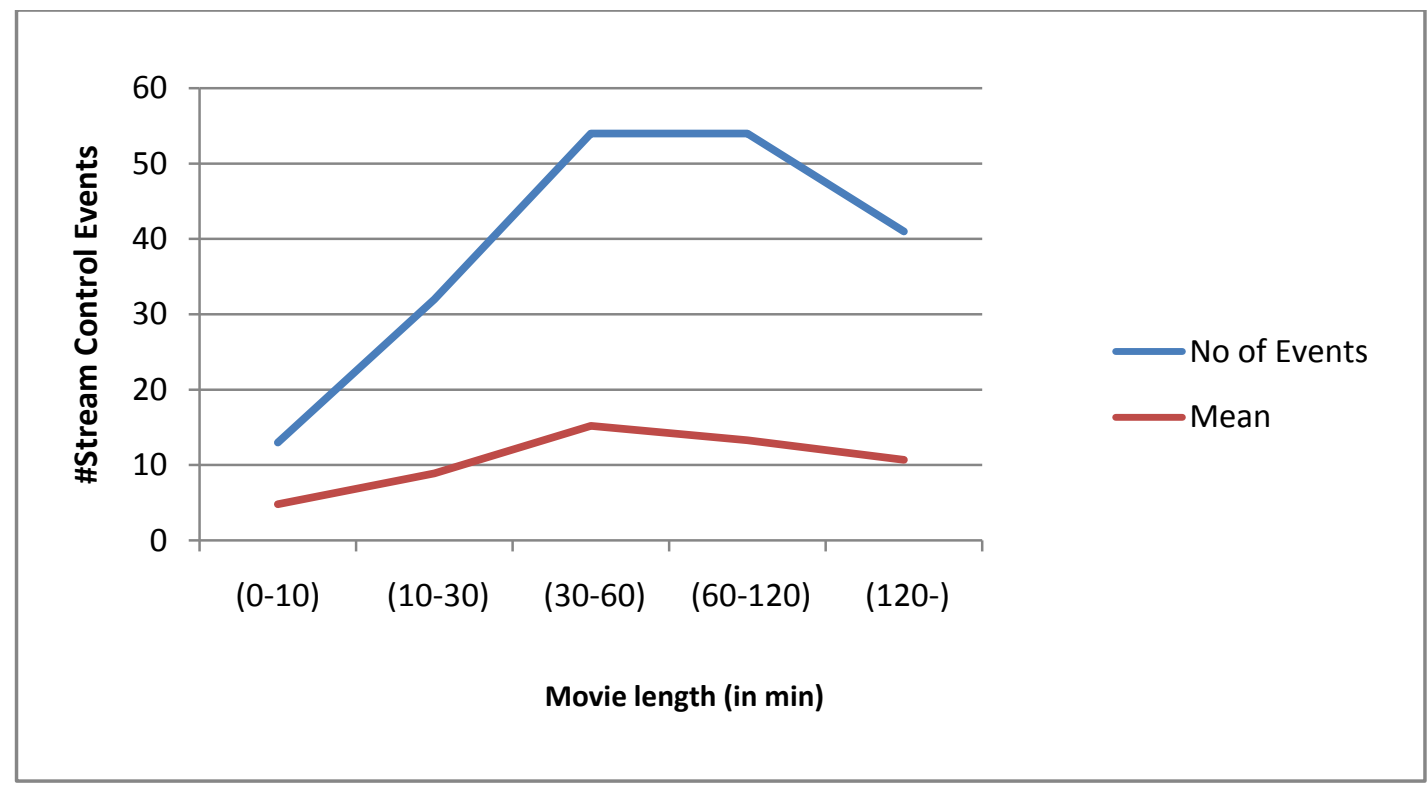

Figure 8 - \# of events for a session vs. movie length (Weekday)

As shown in Figure 8, it is evident that the number of events in user session by movie length. The results revealed that the number of events changed as movie progresses. These results are captured from the weekend usage of IPTV. When week end and week day behavior of customers is considered, the number of events in the weekends is almost near that of week day.

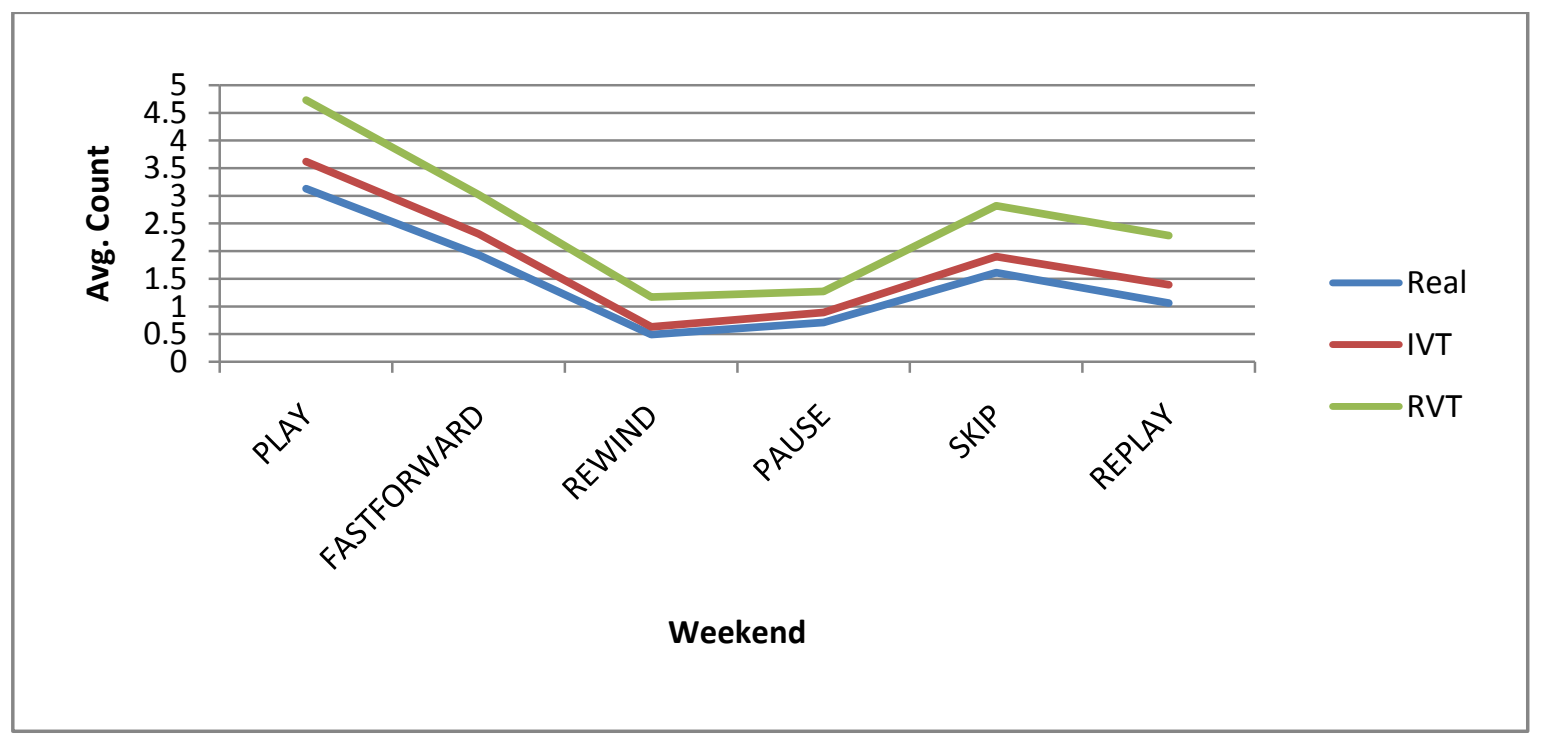

Figure 9 - Stream control event wise breakup of events (Weekend Analysis)

As shown in Figure 9, the stream control events are presented in horizontal axis while the vertical axis represents the average count of events. The user behaviour is captured in the form of the number of occurrences of events that reflect how they were interactively watches VoD through IPTV. 


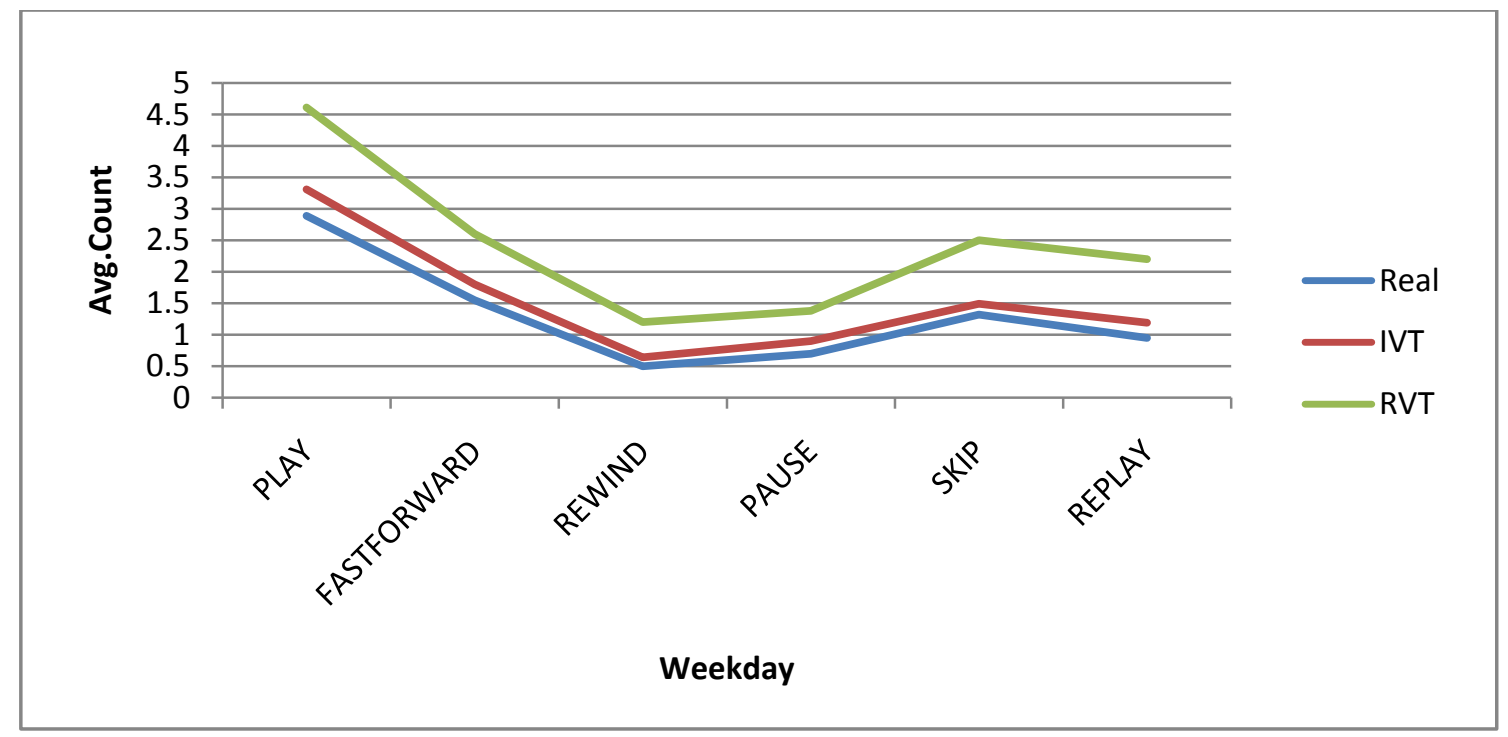

Figure 10 - Stream control event wise breakup of events (Weekday Analysis)

As shown in Figure 10, the stream control events are presented in horizontal axis while the vertical axis represents the average count of events. The user behaviour is captured in the form of the number of occurrences of events that reflect how they were interactively watches VoD through IPTV. The results were related to weekday behaviour of IPTV users.

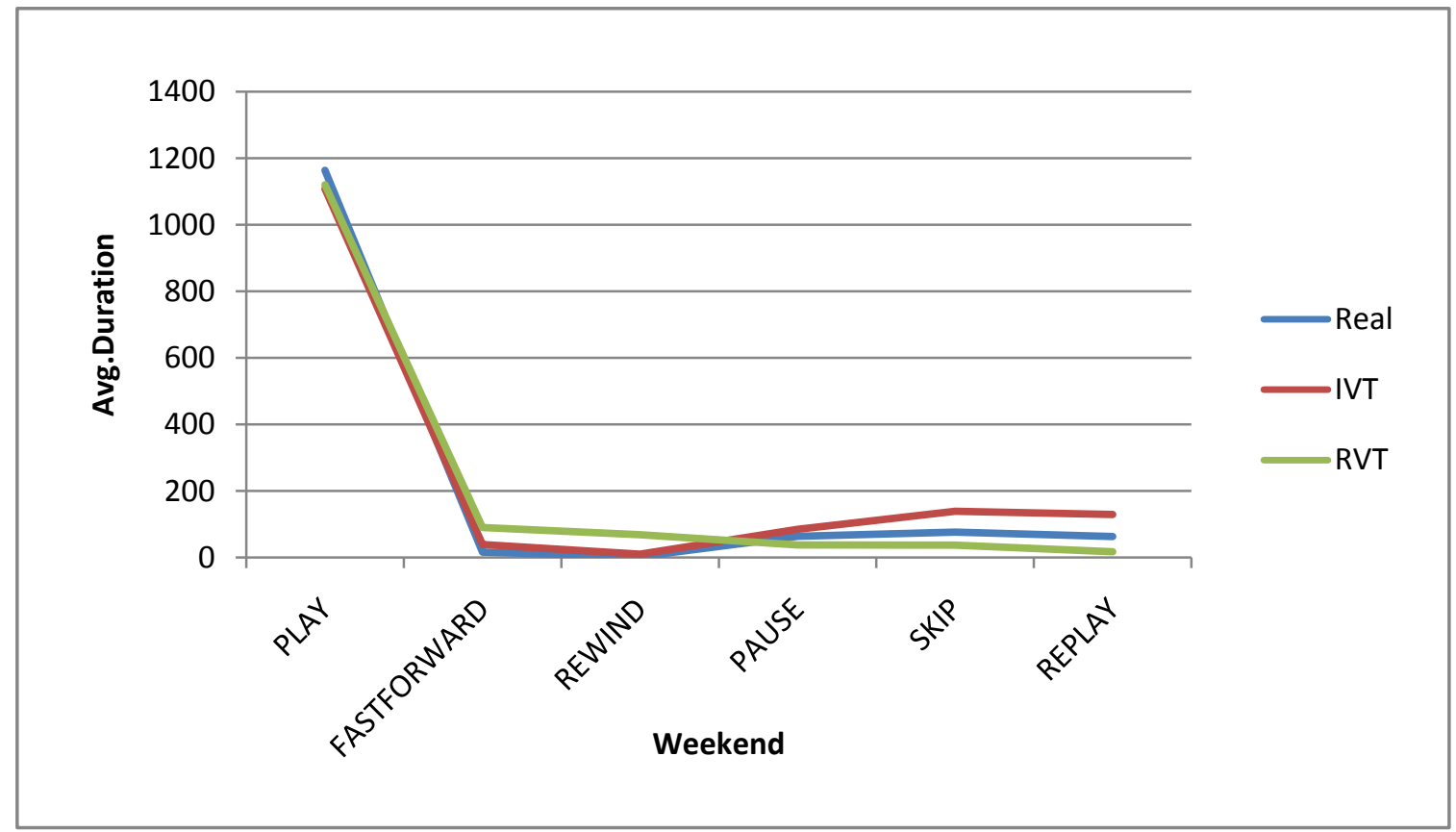

Figure 11 - Stream control event wise breakup of events (Weekend Analysis)

As shown in Figure 11, the stream control events are presented in horizontal axis while the vertical axis represents the average count of events. The user behaviour is captured in the form of the average duration of events that reflect how they were interactively watches VoD through IPTV. The results were related to weekend behaviour of IPTV users. 


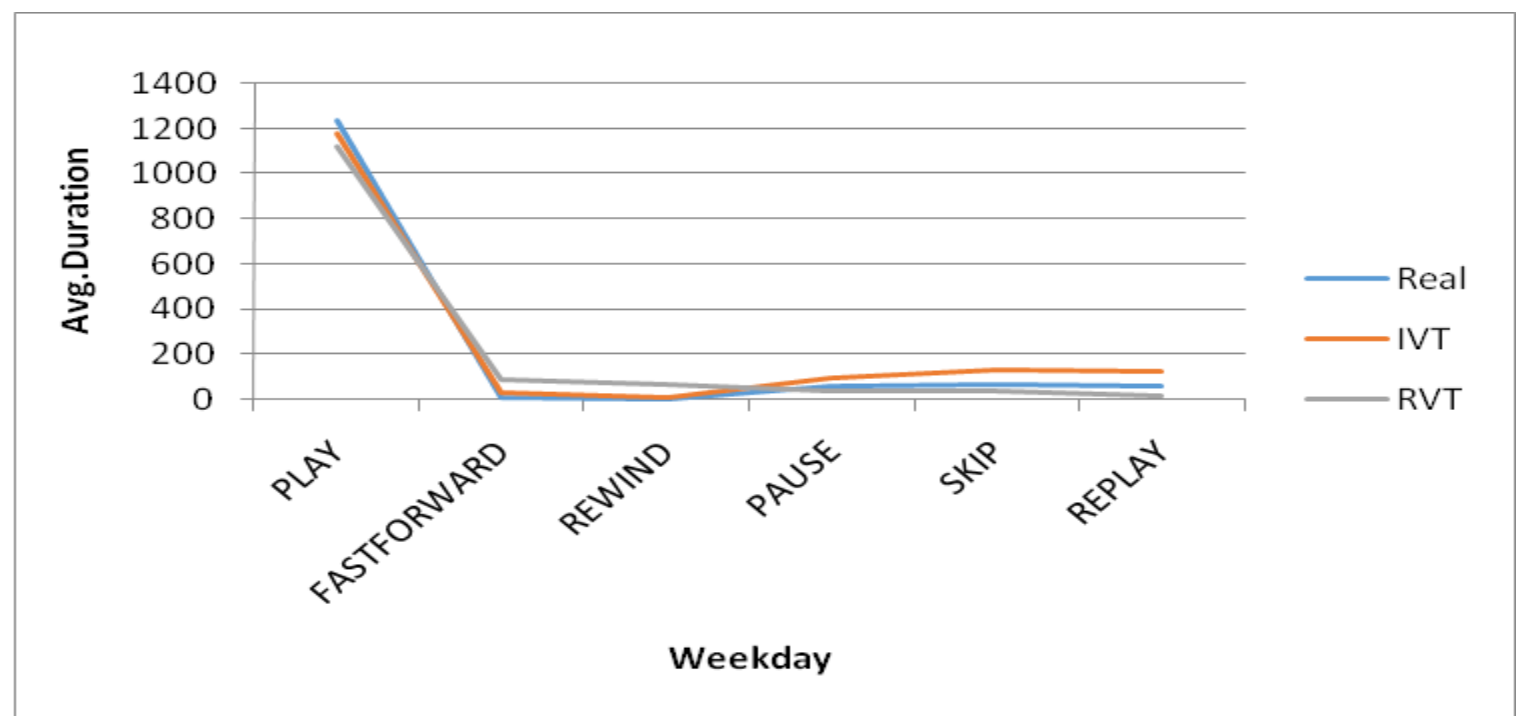

Figure 12 - Stream control event wise breakup of events (Weekday Analysis)

As shown in Figure 12, the stream control events are presented in horizontal axis while the vertical axis represents the average count of events. The user behaviour is captured in the form of the average duration of events that reflect how they were interactively watches VoD through IPTV. The results were related to weekday behaviour of IPTV users.

\section{CONCLUSIONS AND FUTURE WORK}

In this paper, the usefulness of modeling the user behavior of IPTV has been studied with respect to interactive VoD usage. As the subscribers are in large number for IPTV, the users' behaviour with respect to streaming control events such as stop, no action, pause, replay, skip, rewind, fast forward, play, exit and start. User interactivity is modelled based on the traces reflected in the data. An arrival process is modeled that can capture the frequency and modelling periodic bursts. The stream control model is split into two parts. They are known as independent visit time and restricted visit time. These two models are used to model the stream control events aforementioned. The data collected and used is of two years' traces of behaviour of IPTV users. In all the observations play time is more when compared with other streaming control events. The average count and the average duration of events for week end and week day revealed many insights. The average count in the week end for fast forward and skip events is more when compared with other events. With respect to average duration skip and replay durations are more. When the approaches are compared the RVT exhibited more duration in weekday while the same showed least average count. Average count is relatively more in week end when compared with that of weekday. Similarly the average duration is also in weekend is more when compared with that of weekday. The research can be extended further by considering the integration of the proposed models with expectation confirmation models to study users' continuance intention.

\section{REFERENCES}

[1] Tongqing Qiu,Zihui Ge,Seungjoon Lee. (2009). Modeling User Activities in a Large IPTV System. ACM, p.45-56.

[2] Tung-Ching Lin a, Sheng Wu b, Jack Shih-Chieh Hsu a, Yi-Ching Chou. (2012). The integration of value-based adoption and expectation-confirmation models: An example of IPTV continuance intention. Elsevier. 54, p.20-30.

[3] Harry Bouwman, Meng Zhengjia, Patrick van der Duin and Sander Limonard. (2008). A business model for IPTV service: a dynamic framework. ACM, p.20-30.

[4] Cristina Hava Muntean,Gabriel-Miro Muntean. (2005). Framework for Interactive Personalised IPTV for Entertainment. ACM, p.12-19.

[5] Yonghee Shin , Hyori Jeon, Munkee Choi. (2008). Analysis on the Mobil IPTV Adoption and Moderator Effect Using Extended TAM Model.IEEE, p.45-56.

[6] Jun Kyun Choi, Gyu Myoung Lee and Hyo Jin Park. (2008). Web-based Personalized IPTV Services over NGN. IEEE, p.901-1002.

[7] Dong Hee Shin. (2007). Potential user factors driving adoption of IPTV. What are customers expecting from IPTV. Elsevier. 74, p.45-56.

[8] Jongwoo Kim \& Sanggil Kang. (2013). An ontologybased personalized target advertisement system on interactive TV. Springer-Verlag London Limited, p.2030.

[9] Matej Zajc, Kemal Alič, Irena Battelino, Jurij Tasič. (2006). Challenges of Interactive Digital Television for tLearning. ACM, p.12-19.

[10] Pat Diminico, Vijay Gopalakrishnan, Rittwik Jana, K.K. Ramakrishnan. (2011). Capacity Requirements for OnDemand IPTV Services. IEEE, p.901-1002.

[11] [Thomas Silverston, Olivier Fourmaux , Alessio Botta , Alberto Dainotti , Antonio Pescapé , Giorgio Ventre , Kavé Salamatian. (2009). Traffic analysis of peer-to-peer IPTV communities. Elsevier, p.20-30.

[12] Vijay Gopalakrishnan, Rittwik Jana, K. K. Ramakrishnan. (2011). Understanding Couch Potatoes: Measurement and Modeling of Interactive Usage of IPTV at large scale. ACM, p.901-1002. 
[13] Christian Riede,Oliver Friedrich, Robert Seeliger, Stefan Arbanowski. (2008). Interactive IMS-based IPTV. ACM, p.90-101.

[14] Jo Groebel. (2009). Internet Television. ACM, p.20-30.

[15] João Benedito dos Santos Junior,Iran Calixto Abrão, Eduardo Barrére,Paulo Muniz de Ávila, Gabriel Massote Prado. (2008). A Platform for Difusion Interactive Multimedia Content: An Approach Focused on IPTV System and Broadcasting Digital Television System.Elsevier, p.80-86.

[16] Seung-Bum Lee, Student Member, IEEE, Gabriel-Miro Muntean, Member, IEEE, and Alan F. Smeaton, Member, IE. (2009). Performance-Aware Replication of Distributed Pre-Recorded IPTV Content. IEEE, p.12-19.
[17] Mingshan Ma, Yanyan Ma, Jianbo Wang. (2008). The Implementation and Application of IPTV Supported on Pull Mode of P2P.International Symposium on Knowledge Acquisition and Modeling, p.45-56.

[18] Ali C. Begen, Cisco. (2010). On the Use of RTP for Monitoring and Fault Isolation in IPTV. IEEE, p.12-19.

[19] Matthias W. Kampmann. (2011). Predicting IPTV usage: An SEM Approach. ACM, p.45-56.

[20] Geena Shin, Joong-Ho Ahh, Taeha Kim. (2013). IPTV in Korea: The Effect of Perceived Interactivity on Trust, Emotion, and Continuous Use Intention. Asia Pacific Journal of Information Systems. 23 (3), p.20-30 